\begin{tabular}{|c|} 
Clinico- \\
pathological \\
conference
\end{tabular}

Department of

Genitourinary

Medicine, King's

College Hospital NHS

Trust, London

SE5 9RS

L Greene

A L Pozniak

\section{Departments of Genitourinary \\ Medicine and \\ Histopathology, \\ UMDS, St Thomas's \\ Hospital, London \\ SE1 7EH \\ B Peters \\ S B Lucas}

Correspondence to: Dr A L Pozniak, St Stephen's Centre, Chelsea and Westminster Hospital, 369 Fulham Road, London SW10 9TH

Accepted for publication 19 January 2000

\title{
Extrapulmonary tuberculosis masking disseminated histoplasmosis in AIDS
}

\author{
L Greene, B Peters, S B Lucas, A L Pozniak
}

\section{Case presentation (Dr L Greene and Dr A L Pozniak)}

A 32 year old black African man was admitted from our emergency clinic complaining of right sided abdominal pain which was dull in nature with colicky exacerbations that had been present for 6 weeks. The pain had increased in severity over the preceding 3 days. The patient also reported watery diarrhoea and weight loss of $7 \mathrm{~kg}$ over the same period.

He had been found to be HIV antibody positive 1 month previously following an episode of shingles. He reported no risk factors for HIV infection other than unprotected vaginal intercourse in Africa up to 7 years previously. He had no other significant medical history and had not travelled outside of Europe since leaving west Africa in 1990. His CD4 count at diagnosis was less than 20 cells $\times 10^{6} / 1$. His only medication at presentation was co-trimoxazole $960 \mathrm{mg}$ three times weekly as primary prophylaxis against Pneumocysitis carinii pneumonia and co-proxamol for pain relief.

On examination he was thin and mildly pyrexial at $37.5^{\circ} \mathrm{C}$. Scarring from his recent attack of shingles was present on the left anterior thigh in the distribution of the L2 dermatome. The oral cavity was clear. Bilaterally, shotty, non-tender lymph nodes were present in the cervical, axillary, and inguinal regions. There were no cardiovascular or respiratory abnormalities. In the abdomen he had a large, tender, irregular non-mobile right iliac fossa mass that was dull to percussion which extended from the pelvis to below the level of the umbilicus. No other masses were palpable. Neurological examination was unremarkable.

Baseline investigations showed the haemoglobin was $10.7 \mathrm{~g} / \mathrm{dl}$, total white cell count was $3.2 \times 10^{9} / 1$, and platelet count was $122 \times 10^{9} / 1$. The mean cell volume was low at $75 \mathrm{fl}$ with a ferritin level within normal limits. The aspartate aminotransferase (AST) and albumin were abnormal at $75 \mathrm{IU} / 1$ (normal range 0-37 IU/1) and $29 \mathrm{~g} / 1$ (normal range 33-47 g/l) respectively, and the rest of the blood biochemistry was otherwise unremarkable. Bacterial and mycobacterial blood cultures, serum cryptococcal antigen, and blood, urine, and throat specimens for cytomegalovirus early antigen detection were all performed. Stool was examined for the presence of bacterial pathogens, ova cysts and parasites, and Clostridium difficile toxin. Cysts of Cryptosporidium parvum were detected, but all other microbiological investigations were negative.

A chest radiograph was normal. An abnormal bowel gas pattern in the region of the mass was seen on a supine abdominal film. A computed tomograph (CT) scan of the abdomen and pelvis demonstrated a mass apparently comprising matted loops of distal ileum and lymph nodes (fig 1). Hepatomegaly, moderate splenomegaly, and mesenteric and retroperitoneal lymphadenopathy were also noted. Barium studies were performed in order to better define the mass. These confirmed that the mass involved the distal ileum and caecum and showed an ileocolic fistula. An ultrasound guided percutaneous needle biopsy of the mass was performed. Histological examination of the material obtained was reported as showing lymph node tissue containing areas of granulomatous inflammation in association with acid and alcohol fast bacilli. The presence of a single yeast was questioned but not confirmed. A CT guided attempt to obtain a further specimen from the mass was unsuccessful.

On the basis of these findings a diagnosis of abdominal tuberculosis was made and antituberculous therapy was initiated with rifampicin, isoniazid, ethambutol, and pyrazinamide in conventional doses. In view of the patient's weight loss and poor caloric intake, a feeding gastrostomy was inserted percutaneously.

Over the course of the following 7 days a marked reduction in the size and tenderness of the abdominal mass was observed which was paralleled by resolution of the patient's fever. He was discharged from hospital and at outpatient review 2 and 4 weeks later, further improvements in symptoms and signs were reported. Eight weeks after discharge the patient reattended the emergency clinic at which time he was complaining of intermittent high fevers, sweats, and watery diarrhoea. He had also developed an itchy rash on his right shoulder. He was adamant that he had adhered to the antituberculous therapy.

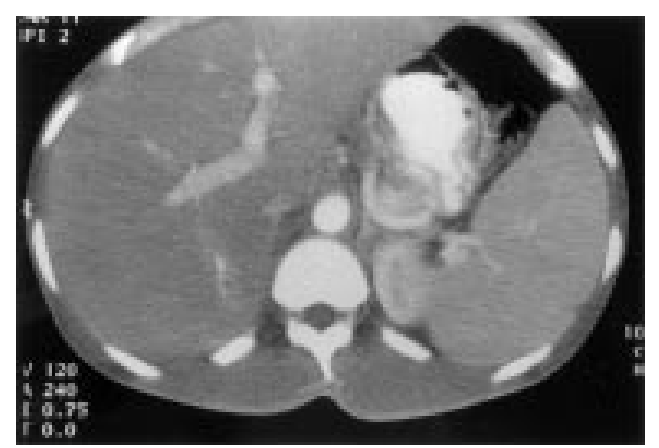

Figure 1 Computed tomograph scan through abdomen (with contrast). Mass of lymphadenopathy involving loops of distal ileum. Note moderate enlargement of the liver and spleen. 


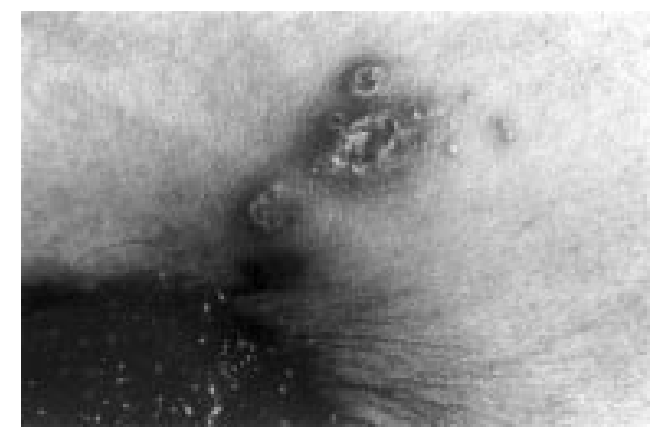

Figure 2 Group of ulcerated inflamed and pigmented papules on arm and neck.

On examination he was markedly pyrexial, temperature $39.5^{\circ} \mathrm{C}$, and he had lost $3 \mathrm{~kg}$ in weight since his last clinic visit. A papular rash with lesions resembling molluscum contagiosum, some of which were ulcerated, was present on the medial aspect of the upper right arm and neck (fig 2). There was persistent lymphadenopathy in the distribution previously noted. Cardiovascular and respiratory examination was unremarkable. In the abdomen moderate hepatomegaly and splenomegaly were again present but no other masses were detected.

The patient was readmitted for further investigation. He was found to be pancytopenic, haemoglobin $9.0 \mathrm{~g} / \mathrm{dl}$, total white cell count $0.93 \times 10^{9} / 1$, neutrophil count $0.6 \times 10^{9} / 1$, platelet count was $60 \times 10^{9} / 1$. The AST was raised at $266 \mathrm{IU} / 1$ and serum albumin was low at $24 \mathrm{~g} / 1$. Liver and renal biochemistry was otherwise normal. Blood and stool cultures were repeated and revealed no pathogens. An abdominal CT scan confirmed resolution of the previously detected mass.

Biopsy of the skin lesions and a bone marrow aspirate and trephine were performed. Examination of the aspirate revealed the presence of macrophages laden with capsulated yeast resembling Histoplasma capsulatum (fig 3). A diagnosis of disseminated histoplasmosis was made and treatment with oral itraconazole suspension $300 \mathrm{mg}$ twice a day was begun. Over the first 7 days of treatment an improvement in his clinical condition was observed with a par-

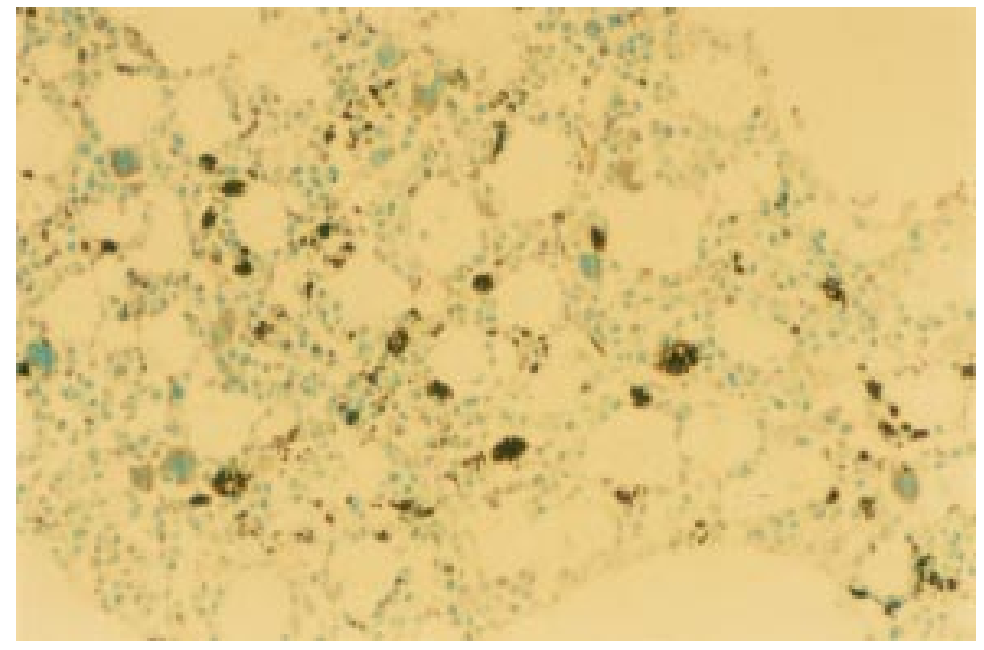

Figure 3 Bone marrow examination showing macrophages laden with capsulated yeasts.

\section{Key points}

- HIV infected patients with tuberculosis may have other reasons for persistent fever: drug resistant tuberculosis should always be considered as one of them.

- Disseminated histoplasmosis should be treated with amphotericin B.

- Bone marrow examination is a useful diagnostic test in patients with undiagnosed fever. ${ }^{10}$

tial resolution of fever and the full blood count began to normalise.

The patient then became acutely unwell with a dry cough, tachypnoea, and hypoxia (oxygen saturation $=86 \%, \mathrm{PaO}_{2}=8.6 \mathrm{kPa}, \mathrm{PaCO}_{2}=4.2$ $\mathrm{kPa}$, breathing room air), and a temperature of $40^{\circ} \mathrm{C}$. He was again pancytopenic with grossly abnormal liver function tests and deranged clotting studies. A chest radiograph and fibreoptic bronchoscopy with bronchoalveolar lavage were performed and were all normal. Examination of a peripheral blood smear demonstrated numerous yeasts within macrophages. At this point itraconazole was discontinued and intravenous amphotericin was substituted with dose escalation up to $1 \mathrm{mg} / \mathrm{kg}$ daily. The patient improved gradually over the following week and thereafter completed a 6 week course of amphotericin. He remains on itraconazole suspension as maintenance therapy 18 months later.

Final clinical diagnosis:

(1) AIDS

(2) Extrapulmonary tuberculosis

(3) Disseminated histoplasmosis

(4) Cryptosporidiosis.

\section{Discussion (Dr B Peters)}

The most obvious differential diagnosis of an abdominal mass, fever, and anaemia in an HIV infected patient originating from sub-Saharan Africa is tuberculosis. However, lymphoma can cause a similar picture and other mycobacterial disease, particularly Mycobacterium avium complex, must be considered. The physicians confirmed the diagnosis of tuberculosis by biopsy but they were informed there was one yeast visible which resembled histoplasmosis and in retrospect this was important information. ${ }^{12}$ I would have pursued this more aggressively and taken further biopsies and the additional diagnosis of histoplasmosis might then have been made at an earlier stage. Detection of anti-Histoplasma capsulatum antibodies by immunodiffusion or complement fixation is positive in about $70-80 \%$ of cases. It was interesting that the mass in the abdomen completely disappeared with antituberculosis therapy, suggesting that the histoplasma load might have been small at that time.

Then the patient re-presented with fevers and anaemia. A relapse of tuberculosis was considered, perhaps with a multidrug resistant strain. The patient had taken his antituberculous therapy regularly but, because he had concomitant cryptosporidiosis, it was possible that 
as a result of diarrhoea he was not absorbing his antituberculous treatment and this is a risk factor for developing multidrug resistant tuberculosis. ${ }^{3}$ The practicalities of regular drug level monitoring of antituberculous therapy as a routine still need to be resolved. If he had relapsed with tuberculosis I would have expected his abdominal mass to recur and therefore pursuing a second diagnosis was appropriate.

When the histoplasmosis was diagnosed he was treated with itraconazole initially, based on the clinicians' belief that the histoplasma load was small. ${ }^{45}$ However, when $H$ capsulatum was found in the bone marrow and culture of blood, his therapy was appropriately changed to amphotericin B.

Physicians looking after HIV positive patients should always be aware of multiple diagnoses even when patients present with an apparently clear cut diagnosis such as histologically and culture proved tuberculosis. One should always be alert for diseases that may clinically cause a similar picture and that may occur concomitantly even when one of these is being adequately treated. This case report is yet another example of Occam's razor (of diagnostic parsimony) not applying in HIV infected patients.

\section{Pathology (Professor S B Lucas)}

The bone marrow biopsies show the small round budding cells typical of $H$ capsulatum and the buffy coat contained numerous organisms, which are seen in HIV patients with severe disseminated diseases.

The two varieties var capsulatum and var duboisii (also called African histoplasmosis) are indistinguishable in their mycelial form but differ in their parasitic form. The cells of the tissue from var duboisii are much larger and have thicker walls than var capsulatum. Although the patient was from Africa, he had var capsulatum infection.

$H$ capsulatum var capsulatum can, on microscopic examination, be confused with other pathogens such as Penicillium marneffei as well as with atypical Blastomyces dermatitidis and small non-encapsulated Cryptococcus neoformans. One of the patient's skin lesions was caused by herpes simplex and the other was an ulcerated molluscum contagiosum; neither was due to disseminated fungal infection.

\section{Comment (Dr A L Pozniak)}

It is a shame that we did not have access to $H$ capsulatum serum or urine antigen testing when the single yeast was reported as this is an extremely sensitive and specific method for diagnosing histoplasmosis. ${ }^{6}$ One of the major problems in managing this patient was that of drug-drug interactions. His rifampicin had to be changed to rifabutin because (after a washout period) he was started on indinavir. ${ }^{7}$ Other options for antiretroviral therapy are now possible. ${ }^{8}$ Itraconazole and rifampicin given together may result in very low levels of itraconazole. ${ }^{9}$ In this patient as the CD4 count has not risen above 70 cells $\times 10^{6} / 1$, despite an undetectable $(<400$ copies $/ \mathrm{ml}$ ) viral load, the itraconazole has been continued. Once the CD 4 count rises above 200 cells $\times 10^{6} / 1$ it might be possible to discontinue the itraconazole.

This case was presented as an unseen case at King's College School of Medicine in October 1997 when Dr B Peters was the discussant.

We thank Louise Hinds for typing the manuscript and Dr Rob Miller for editing the manuscript.

1 Graybill JR. Histoplasmosis in AIDS. $f$ Infect Dis 1988;158:623-5.

2 Sarosi GA, Johnson PC. Disseminated histoplasmosis in patients with human immunodeficiency virus. Clin Infect Dis 1997; 14:S60-7.

3 Peloquin CA, MacPhee AA, Berning SE. Malabsorption of antimycobacterial medications. N Engl f Med 1993; 329:1122.

4 Wheat LJ, Connolly-Stringfield P, Baker RL, et al. Disseminated histoplasmosis in the acquired immune deficiency syndrome: clinical findings, diagnosis and treatment and syndrome: clinical findings, diagnosis and treatm
review of the literature. Medicine 1990;69:361-74.

5 Wheat LJ, Hafner RE, Ritchie M, et al. Itraconazole is effective treatment for histoplasmosis in AIDS. 31 st ICAAC, Anaheim, USA, 1992 .

6 Wheat LJ, Kohler RB, Tewan RP. Diagnosis of histoplasmosis by detection of Histoplasma capsulatum antigen in serum and urine specimens. N Engl F Med 1986;314:83-8.

7 Centres for Disease Control and Prevention. Clinical update: impact of HIV protease inhibitors on the treatment of HIV infected tuberculosis patients with rifampicin. MMWR 1996;45:921-5.

8 Pozniak AL, Miller R, Ormerod LP. The treatment of tuberculosis in HIV infected persons. AIDS 1999;13:435-45.

9 Heylen R, Miller R. Adverse effects and drug interactions of medications commonly used in the treatment of adult HIV positive patients. Genitourin Med 1996;72:237-46.

10 Brook MG, Ayles H, Harrison C, et al. Diagnostic utility bone marrow sampling in HIV positive patients. Genitourin Med 1997;73:117-21. 